\title{
Current Controversies in Prenatal Diagnosis 1: In utero therapy for spina bifida is ready for endoscopic repair
}

Michael Belfort ${ }^{1}$, Jan Deprest ${ }^{2,3}$ and Kurt Hecher ${ }^{4 \#}$

${ }^{1}$ Department Obstetrics \& Gynecology, Baylor College of Medicine, Texas Medical Center, Houston, Texas, US

${ }^{2}$ Department of Obstetrics \& Gynecology, University Hospitals Leuven and Development \& Regeneration, Biomedical Sciences, KU Leuven, Leuven, Belgium

${ }^{3}$ Research Department of Maternal Fetal Medicine, Institute for Women's Health, University College London, London, UK.

${ }^{4}$ Department of Obstetrics \& Fetal Medicine, University Medical Center, Hamburg-

Eppendorf, Hamburg, Germany

\#Correspondence to: Kurt Hecher. E-mail: k.hecher@uke.de

This written debate summarizes the oral presentations made at the 2016 International Society for Prenatal Diagnosis meeting in Berlin, Germany. It does not necessarily reflect the personal opinions of each of the authors.

All authors contributed equally to this work.

Funding Source: None

Conflicts of Interest: None declared

What's already known about this topic?

- There is level 1 (RCT) evidence for improved neurological outcomes after open fetal surgery for spina bifida vs. postnatal surgery.

- There is a lack of standardization of techniques and comparative outcome data in clinical trials for endoscopic fetal surgery for spina bifida.

What does this study add?

- Different techniques, their strengths, limitations and clinical implications of endoscopic vs. open fetal surgery for spina bifida are discussed.

- Open fetal surgery anticipates postnatal surgery, whereas novel and less invasive endoscopic approaches aim to simplify the technique.

- More data on long-term follow up of intrauterine closure of spina bifida are needed and open surgery is one step ahead of fetoscopic approaches.

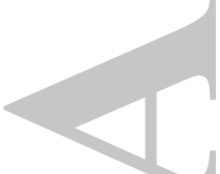

This article has been accepted for publication and undergone full peer review but has not been through the copyediting, typesetting, pagination and proofreading process which may lead to differences between this version and the Version of Record. Please cite this article as doi: $10.1002 / p d .4972$ 
INTRODUCTION (KURT HECHER)

The fetus has become a potential patient and intrauterine surgery is a realistic option for fetal therapy under certain circumstances. Its aim is to prevent intrauterine fetal death or fetal damage owing to fetal disease for which postnatal therapy or surgery comes too late. Nowadays, the most frequently performed intrauterine surgery is fetoscopic laser ablation of placental vascular anastomoses in monochorionic twin pregnancies with severe twin-totwin transfusion syndrome. If left untreated, this has a mortality of $80-90 \%$ and laser coagulation destroys the underlying prerequisite for the syndrome by converting a hemodynamically monochorionic into a dichorionic placenta. This leads to a significant reduction in mortality and long-term morbidity of affected fetuses. Indications for fetal surgery are severe congenital diaphragmatic hernia $(\mathrm{CDH})$ and spina bifida or myelomeningocele (MMC). There are three major differences between these two malformations. First, in severe $\mathrm{CDH}$ there is a high mortality owing to pulmonary hypoplasia and hypertension in the newborn, whereas MMC is a non-lethal malformation leading to lifelong neurological impairment of lower limb motor and sensory functions, incontinence and hindbrain herniation causing hydrocephalus, which may have to be treated postnatally by a ventriculo-peritoneal shunt. Second, $\mathrm{CDH}$ can be effectively treated by minimally invasive fetoscopic balloon occlusion of the trachea, which may lead to significant lung growth, whereas the fetoscopic approach is much more difficult in MMC, where complete closure of the skin defect must be achieved to avoid damage of the exposed neural tissue. Third, a multicenter randomized controlled trial (www.totaltrial.eu) to compare fetoscopic intrauterine surgery with postnatal surgery in $\mathrm{CDH}$ is currently in progress, whereas fetoscopic intrauterine surgery for MMC has been abandoned after small initial trials with rather sobering results. ${ }^{1-4}$ However, a RCT comparing open fetal surgery with postnatal neurosurgical repair, the management of myelomeningocele study [MOMS trial], reported improved short-term neurological outcomes in the fetal surgery group. ${ }^{5}$ Only recently a renaissance in fetoscopic surgery for MMC has occurred with several centers reporting new techniques and promising short-term results. From a maternal point of view one would expect fewer complications, whereas from a fetal point of view the question is whether the outcome is as good as for open fetal surgery. Therefore, the question for this debate was whether in utero therapy for spina bifida is ready for fetoscopic repair. 
Fetoscopic surgery for the minimally invasive repair of open neural tube defects (ONTD) was pioneered by Bruner and Tulipan ${ }^{1-3}$ and by Farmer et al $^{4}$ from San Francisco. Results were disappointing with a high perinatal mortality rate, and no further research into fetoscopic approaches occurred for almost 2 decades in the USA. Instead, an open hysterotomy approach was developed and studied at the 3 centers in the MOMs trial. ${ }^{5}$ Closure techniques at the 3 centers varied depending on the lesion size and surgeon and details about how the closure technique influenced the outcomes has not been reported. In Philadelphia, a complex 3 layer repair technique that used extensive dissection, myofascial flaps, and at times patches to effect a watertight closure is recommended, however, the best repair has yet to be determined. In Europe however, Thomas Kohl developed a completely percutaneous three to five port fetoscopic technique with an extensive dissection and patch repair. ${ }^{6-8}$ Early outcomes were not well accepted by the fetal surgery community because of the high rate of preterm delivery and because long term neurological outcomes were not available. ${ }^{9,10}$ In Brazil, Pedreira et al. ${ }^{11-13}$ also developed a percutaneous fetoscopic patch repair. Results from these two groups have been progressively improving but detractors still point out that compared with MOMS data, fetoscopic repair is still associated with a higher rate of prematurity and premature rupture of the membranes (PPROM) at less than 37 weeks, (Pedreira et $\mathrm{al}^{13}: 100 \%$ PPROM (10/10) at 30+/-3 weeks; Degenhardt et $a l^{8}:$ 84.3\% PPROM (43/51) at 30+/- 3 weeks; Adzick et $\left.\mathrm{al}^{5}: 46 \%(36 / 78)\right)$, a higher perinatal mortality rate (Pedreira et al ${ }^{13}: 20 \%-2 / 10$; Degenhardt et $\mathrm{al}^{8}: 8 \%-4 / 51$; Adzick et $\mathrm{al}^{5}: 3 \%-2 / 78$ ) and less than equivalent neurological outcomes (need for revision of the repair with additional postnatal surgery ((Pedreira et $\mathrm{al}^{13}: 29 \%(2 / 7)$; Degenhardt et al ${ }^{8}$ : not reported; Adzick et $\left.\mathrm{al}^{5}: 3 \%-2 / 77\right) .{ }^{14}$

Our group, in collaboration with a group in Barcelona, developed an open abdomen exteriorized uterus single port technique in $\mathrm{CO}^{2}$ in a sheep model using a patch and glue approach. ${ }^{15}$ For our human cases, however, we opted to pursue an exteriorized uterus two port in $\mathrm{CO}^{2}$ technique and a simplified neurosurgical repair without patches or glue. ${ }^{16,17}$ Given that there are as many techniques being used as there are groups engaged in researching this type of surgery, any attempt to lump all of the results together in a meta- 
analysis comparing fetoscopy with open repair may be difficult and potentially

misleading. ${ }^{18}$ My statements in this debate pertain to the technique developed at Texas Children's Fetal Center, which differs materially from the fetosocopic techniques of others in its (1) exteriorized uterus approach, (2) its use of humidified and warmed $\mathrm{CO}^{2},(3)$ the plication of the membranes to the uterine wall, and (4) a very different neurosurgical repair. To attempt to lump our data with those of others would not be a fair representation of the field in general, especially when comparing rates of preterm premature rupture of membranes (PPROM), preterm delivery, perinatal mortality and neurological outcomes.

There are 3 outcomes of interest that must be considered:

(1) Maternal outcomes: We have previously shown that maternal outcomes after a fetoscopic repair are similar to those performed using a hysterotomy except for a significantly higher rate of vaginal delivery in the fetoscopy group. ${ }^{17}$ It is difficult to argue that open fetal surgery is safer for the mother given the obvious risks of a $7-10 \mathrm{~cm}$ hysterotomy. These include: bleeding at the time of surgery, uterine rupture, and need for cesarean section in the index and all future pregnancies, and the potential for placenta percreta in future pregnancies. The option of a vaginal delivery at term is also a paradigm shifting distinction between the two approaches and cannot be ignored. From a maternal aspect, we have to agree that fetoscopy is a safer and better option.

(2) Fetal and Neonatal non-neurological outcomes: Our fetoscopic patients (Table 1) did comparably well with our open surgery patients with no higher rates of prematurity, neonatal depression, or any other non-neurological complication. ${ }^{17}$ Given that we are in the process of publishing all of our results I cannot be specific here, but in our latest cohort with a standardized technique, the mean gestational age at delivery is now well into the term range, with a less than $20 \%$ PPROM rate and a more than $50 \%$ rate of vaginal delivery. While we have to acknowledge that numbers are small and our fetoscopic group sample size is not equal to that of either our open group or that of the MOMS group ${ }^{5}$, and that these are comparisons of uncontrolled, non-randomized patients, we also have to accept that all of these patients were selected based on the same MOMS criteria and managed pre- and postoperatively using the same or a similar protocol based on the published MOMS methodology. ${ }^{5}$ The strength of the comparison lies in the fact that we are comparing the 
results of the fetoscopic and open approaches of a single group (Texas Children's Fetal Center) with the results of the open approach from the MOMS trial. ${ }^{5}$ This removes the variability introduced by trying to compare the immediate neonatal results of 3 different fetoscopic approaches with different rates of PPROM, oligohydramnios, preterm delivery, gestational age at the time of delivery, and perinatal mortality from prematurity. ${ }^{6-8,11-}$ 13,16,17 In my opinion this second issue is a qualified one: for our technique I am confident in saying that non-neurological outcome results are comparable between our fetoscopic and open cases and between our fetoscopic cases and those reported in the MOMS trial. ${ }^{5} \mathrm{I}$ am not prepared to comment on whether the entire field, when data are aggregated, can demonstrate the results that we are seeing. I do believe that as we all gain experience in our respective techniques, and as we work together to develop a more standardized technique that can be rigorously tested against the open approach, we will show comparable results in non-neurologic outcomes.

(3) Neurological outcome: A reasonable comparison between open and fetoscopic approaches is currently impossible as long as we are all performing very different repairs. Professor Deprest supports an extensive dissection of the defect into its separate layers followed by a stepwise closure utilizing multiple layers of suture material, the development of significant myofascial flaps, and when necessary patches to effect a watertight closure. In my opinion, a simple closure utilizing the available tissue with no extensive dissection (which limits bleeding, release of inflammatory mediators, and inevitable invasion of fibroblasts and scar tissue) is a more logical approach. Our technique creates a natural covering of the placode that we believe will heal with minimal inflammation and scarring. Of course at this time our data, although encouraging, are not robust enough for meaningful comparison with the MOMS dataset. ${ }^{5}$ We will likely need a decade to prove our point and in this respect my opponent has an advantage in being able to quote medium term and long term MOMS outcome data. Our technique (and likely those of my compatriots in the fetoscopic world) is still improving, and those of us performing fetoscopic open neural tube defect repair are just exiting our learning curves. Our team has already noted a decrease in leakage of CSF at birth and better reversal of hindbrain herniation rates in our latest cases when compared with our initial ones. We are seeing early neurological outcomes that are equivalent to our open cases and equivalent to the published MOMS data. ${ }^{5}$ Given that we 
have a paper in preparation I am unable to go into specific details here. Long term outcome on the neurodevelopmental, behavioral, cognitive, functional motor, and bladder and sexual function of this cohort of people is still developing and is crucially important to collect and report.

Thus, I hope to have convinced the audience that (1) from a maternal aspect there can be no question of the benefit of the fetoscopic approach, (2) the neonatal non-neurologic data are suggestive of equivalency for the two techniques, and (3) while it is too early to compare the long-term neurological outcomes, our initial data, as assessed and independently reviewed by our Institutional Review Board, our Data Safety and Monitoring Board, the Federal Drug Administration, and our Fetal Therapy Board, are promising enough to allow continued investigation of this methodology. I am certain that both Dr. Deprest and I can agree on this - that as we go forward we all need to collect the same data, using the same tests and criteria, in the same international registry, and then compare our outcomes with complete transparency to the benefit of our maternal, fetal and pediatric patients.

\section{AGAINST (JAN DEPREST)}

The natural history of most forms of spinal dysraphism shows progression in utero. As a consequence, fetal surgery was conceived to arrest the second of two pathogenetic "hits", i.e. acquired damage to the exposed cord and nerves, ${ }^{19-21}$ and cerebrospinal fluid leakage, acting as a suction gradient and causing a Chiari II Malformation (CM) and/or ventriculomegaly. ${ }^{20,22-25}$ The experimental basis for the procedure, which we currently offer in Leuven, essentially consists of the demonstration in animal models that timely and complete coverage leads to in utero reversal of the $\mathrm{CM}$ and rescue of neurologic function. ${ }^{21,26}$ Technically, one mimics what is done postnatally, i.e. dissection of the placode followed by either a multi-layered repair, or in the absence of sufficient tissue, using graft materials. After a few initial fetoscopic attempts, the pioneers of this approach resorted to microneurosurgical techniques made possible thanks to broad access to the fetal spine. This required hysterotomy, laparotomy and general maternal anesthesia. 
Because surgery for this non-lethal condition also proved to incur significant maternal and fetal risks, our team only considered offering it after completion of the randomized controlled MOMS trial. ${ }^{5}$ The choice for what is called open materno-fetal surgery (OMFS) was not an obvious one for a team with a tradition for developing fetoscopic instruments and novel fetal surgical techniques or strategies. ${ }^{27}$ Furthermore, our experience to date demonstrates that less than half of patients we evaluate, even after they have opted to continue their pregnancy and traveled long distances to learn about fetal surgery, proceeds with the procedure. ${ }^{28}$ There is no doubt that reducing the invasiveness of the surgery would increase its uptake among mothers, families and physicians; yet why have we not done so?

Today, the surgical correction of the cystic (myelomeningocele) and non-cystic variant (myeloschisis) of open spina bifida consists of a stepwise formal dissection of the placode at the zona epithelio-serosa, untethering of the filum terminale (neurulation is not consistently done), ideally followed by a watertight, layered coverage of the cord using the dura, paraspinal muscles and fascia and finally the skin (Figure 1a). There is no doubt that this is tremendously challenging, if not impossible with the current fetoscopic equipment and expertise. From a neurosurgical viewpoint, following dissection of the placode, fetoscopy does not even attempt primary dural closure and myelofascial coverage. Fetoscopic pioneers understandably resorted to alternatives either using tissue substitutes to cover the spinal cord at the level of the paraspinal muscles or the skin, or both combined (Figures $1 \mathrm{~b}$ and 1c), or basically only closed the skin primarily. Even that is quite challenging, with (initial) failure rates as high as $15 \%$ or more. ${ }^{9,12}$

The above information demonstrates that current fetoscopic operations are not standardized, so that it is difficult to assess the efficacy of fetoscopic repair. Yet, we cannot assume that one or more of the current fetoscopic techniques offer a neuroprotective effect that is equal to the open technique. As watertight closure is a conditio sine qua non for success, we feel that additional experimental validation of the fetoscopic approach in sufficiently powered lamb studies (early induction of a sufficiently large defect, including myelotomy, appropriate interval till repair, comprehensive neonatal imaging, neurophysiologic and histological testing and assessment of adhesions to the cord) is required. This is definitely not the case for the most recent technique proposed by my opponent. Closure of only the skin is a method which, to my knowledge, has neither 
experimentally nor clinically been assessed before ${ }^{16}$ Admittedly, this type of experiments are even relevant to OFMS, as in around $20 \%$ of open cases, graft materials are needed either at the level of the dura or the skin. ${ }^{29}$ Graft augmented surgery has been described in experimental series, yet their designs can be criticized. We are therefore currently exploring all of these technical aspects in our laboratory, yet we have not moved to their clinical implementation.

Apart from the required animal experimental work, clinical fetoscopic programs are in operation, and gather very important clinical data. Their experiences should be meticulously documented in the same format as was and is currently being done for OMFS. ${ }^{30} \mathrm{I}$ am sure that my opponent's co-workers are doing that. However there will come a day when the most commonly performed fetosocpic closure technique will need to be formally compared to standard anatomical repair in a clinical research study.

Fetoscopic repair is not yet standardized and all techniques require insertion of at least two and up to five cannulas. Furthermore, its complexity leads to at least two times longer operation time. Reported intra-uterine fetal death rates exceed those of open repairs. It is also disappointing that minimally invasive spina bifida repair does not reduce the risk for membrane rupture or oligohydramnios. As a consequence, the risk for preterm delivery is not reduced and this may eventually offset the fetal therapeutic effects. ${ }^{18}$

In summary, fetoscopic coverage still requires general maternal anesthesia and creation of sufficient workspace. It remains unclear whether that space is best obtained following uterine exposure, ${ }^{16}$ or percutaneously. ${ }^{9,12,13,31}$ Therefore, minimally invasive spina bifida repair keeps on being a maternal burden. However, we are the first to acknowledge that avoidance of a hysterotomy, which compromises the index and future pregnancies, would be a tremendous step forward. Though no long-term data are available today, it is very likely that multiple cannulation of the pregnant uterus will have little or no effect on future fertility and gynecological outcome. It seems fair to extrapolate that from follow up studies on single cannula fetoscopic surgery for other anomalies. ${ }^{32}$

Families, however, undertake these surgeries with the primary goal of improving outcome of spina bifida. Therefore, no technical modifications are justifiable that would compromise the fetal outcome. It is our belief today that the available peer reviewed short term data do not support equivalence of both approaches. Given the additional lack of properly

This article is protected by copyright. All rights reserved. 
controlled mid-term outcome data, fetoscopic spina bifida repair should be considered as an investigational procedure and cannot surf on the findings of the MOMS trial.

CONCLUSIONS (KURT HECHER)

Both debaters showed clearly the advantages and limitations of the endoscopic technique and the open surgery by hysterotomy approach, respectively, taking into account maternal and fetal benefits and risks. It has to be noted, that Dr. Belfort and his group's open abdomen exteriorized uterus two-port approach is different from that of other groups using a percutaneous fetoscopic technique. Performing a laparotomy before finally selecting the entry point for the fetoscope and another working port may help to gain optimal access to the surgical field and thereby create sufficient working space. Furthermore, the fetoscopic and the open approaches are different regarding the closure techniques. The endoscopic closure is performed with a unified layer of undissected dura, in contrast to many other endoscopic techniques without the application of patches or glues. The open closure technique consists of a multilayered repair that includes the dura, musculofascial structures and the skin after dissection of the placode and untethering of the filum terminale, thereby imitating and anticipating the postnatal technique. Dr. Belfort argues that his approach is simpler and less traumatic and may, therefore, cause less inflammation and scarring. Both debaters agree that a watertight closure is the primary aim of intrauterine surgery, otherwise the protective effect against damage of the exposed spinal cord cannot be expected. Whether this can be achieved equally effective by both approaches still remains to be shown by comparative studies.

Dr. Belfort focuses on maternal, fetal and neonatal non-neurological and early neurological outcomes. For the mother, open fetal surgery has clearly higher risks owing to hysterotomy, which affects the index and all future pregnancies with the need for cesarean section, while the endoscopic approach allows vaginal delivery. Furthermore, bleeding, uterine rupture and morbidly adherent placenta are typical risks after hysterotomy. Dr. Deprest agrees that avoidance of hysterotomy would be a tremendous step forward, if the endoscopic surgery can be done as effectively as the open one. He rightly points out that woman agree to and 
bear the burden of this surgery under the condition that the primary aim of it, namely an improvement of long term morbidity of their child, is achieved, as this is not a lethal malformation, for which a live saving intervention would be indicated. Dr. Belfort confirms that fetoscopic surgeons are just exiting the steeper slopes of growing experience of their learning curves and early neurological outcomes seem to be equivalent to the published MOMS trial data. However, sufficient long-term outcome data are not yet available.

In contrast to endoscopic surgery, long term outcome data are available for open surgery. A recent study reported long term data on neurofunctional outcome from a pre MOMS population with open repair. ${ }^{33}$ At a median age of ten years, $79 \%$ were community ambulators and only $14 \%$ were wheelchair dependent. More than expected were continent, but bowel and bladder control were still ongoing challenges. Abnormalities of behavioral adaptive skills were more common than impairments in executive functioning, and they were associated with the need for shunting.

Taking into account the variety of fetoscopic techniques with insertion of two up to five cannulas and the potential technical difficulties leading to a considerably longer time of surgery needed for endoscopic repair, Dr. Deprest calls for more experimental trials and standardization of the techniques. After this has been achieved, there will be an urgent need for a randomized controlled trial to compare neurological outcomes with those after open fetal surgery, with surgery being performed in centers of excellence for each respective technique. In the meantime, proof of concept innovative surgery in animal models followed by human cases may lead to novel methods of patch repair for the open as well as the fetoscopic approach in cases with large defects in which primary closure is difficult. $^{34}$

latrogenic prematurity is still a major complication of all techniques of intrauterine surgery for spina bifida, regardless whether it is done by fetoscopy or hysterotomy. In all major studies, the mean gestational age at delivery is consistently around 34 weeks. One may wonder, whether on its own this may be a major factor leading to improved outcomes, owing to an average reduction of six weeks of exposure to the toxic amniotic fluid. To test this hypothesis, I would propose a randomized controlled trial comparing the most standardized surgical technique, which at the moment is certainly the open approach of the MOMS trial, with conservative management followed by elective cesarean section at 34 
weeks of gestation. A multidisciplinary working group would be needed to investigate whether this would be justified, feasible and ethically approvable.

In conclusion, there is agreement that the strengths of open fetal surgery for spina bifida repair are the standardization of the technique and that it is the only one for which evidence based neurological outcome data in comparison to postnatal surgery are available.

However, the search for an endoscopic technique, which is less invasive for the mother and equally beneficial for the fetus must be pursued and, once standardization has been achieved, rigorously tested in clinical trials. In selected centers, and within the framework of research protocols, in utero therapy for spina bifida is ready for endoscopic repair.

\section{REFERENCES}

1. Bruner JP, Richards WO, Tulipan NB, Arney TL. Endoscopic coverage of fetal myelomeningocele in utero. Am J Obstet Gynecol 1999;180:153-8.

2. Tulipan N, Bruner JP. Myelomeningocele repair in utero: a report of three cases. Pediatric Neurosurgery 1998;28:177-80.

3. Bruner JP, Tulipan NB, Richards WO, et al. In utero repair of myelomeningocele: a comparison of endoscopy and hysterotomy. Fetal Diagn Ther 2000;15:83-8.

4. Farmer DL, von Koch CS, Peacock WJ, et al. In utero repair of myelomeningocele: experimental pathophysiology, initial clinical experience, and outcomes. Archives of Surgery 2003;138:872-8.

5. Adzick NS, Thom EA, Spong CY, et al. A randomized trial of prenatal versus postnatal repair of myelomeningocele. N Engl J Med 2011;364:993-1004.

6. Kohl T, Tchatcheva K, Merz W, et al. Percutaneous fetoscopic patch closure of human spina bifida aperta: advances in fetal surgical techniques may obviate the need for early postnatal neurosurgical intervention. Surg Endosc 2009;23:890-5.

7. Kohl T, Hering R, Heep A, et al. Percutaneous fetoscopic patch coverage of spina bifida aperta in the human--early clinical experience and potential. Fetal Diagn Ther 2006;21:185-93.

8. Degenhardt J, Schurg R, Winarno A, et al. Percutaneous minimally-invasive fetoscopic surgery for spina bifida aperta - Part II Maternal management and outcome. Ultrasound Obstet Gynecol 2014;44:525-31. 
9. Verbeek RJ, Heep A, Maurits NM, et al. Fetal endoscopic myelomeningocele closure preserves segmental neurological function. Dev Med Child Neurol 2012;54:15-22.

10. Shurtleff D. Fetal endoscopic myelomeningocele repair. Dev Med Child Neurol 2012;54:4-5.

11. Pedreira DA, Quintero RA, Acacio GL, et al. Neoskin development in the fetus with the use of a three-layer graft: an animal model for in utero closure of large skin defects. J Mat Fetal Neonat Med 2011;24:1243-8.

12. Pedreira DA, Zanon N, de Sa RA, et al. Fetoscopic single-layer repair of open spina bifida using a cellulose patch: preliminary clinical experience. J Mat Fetal Neonat Med 2014;27:1613-9.

13. Pedreira DA, Zanon N, Nishikuni K, et al. Endoscopic surgery for the antenatal treatment of myelomeningocele: the CECAM trial. Am J Obstet Gynecol 2016;214:111.e1-11.

14. Flake A. Percutaneous minimal-access fetoscopic surgery for myelomeningocele - not so minimal! Ultrasound Obstet Gynecol 2014;44:499-500.

15. Peiro JL, Fontecha CG, Ruano R, et al. Single-Access Fetal Endoscopy (SAFE) for myelomeningocele in sheep model I: amniotic carbon dioxide gas approach. Surgical Endoscopy 2013;27:3835-40.

16. Belfort MA, Whitehead WE, Shamshirsaz AA, et al. Fetoscopic Repair of Meningomyelocele. Obstet Gynecol. 2015;126:881-4.

17. Belfort $M$, Whitehead WE, Shamshirsaz AA, et al. The exteriorized, CO2-filled human uterus: A new surgical space for fetal surgery? Am J Obstet Gynecol 2016;214:S37

18. Joyeux L, Engels AC, Russo FM, et al. Fetoscopic versus open repair for spina bifida aperta: A systematic review of outcomes. Fetal Diagn Ther 2016;39:161-71.

19. Heffez DS, Aryanpur J, Hutchins GM, Freeman JM. The paralysis associated with myelomeningocele: clinical and experimental data implicating a preventable spinal cord injury. Neurosurgery 1990;26:987-92.

20. Stiefel D, Copp AJ, Meuli M. Fetal spina bifida in a mouse model: loss of neural function in utero. J Neurosurg 2007;106(3 Suppl):213-21.

21. Meuli M, Meuli-Simmen C, Hutchins GM, et al. In utero surgery rescues neurological function at birth in sheep with spina bifida. Nat Med 1995;1:342-7.

22. Korenromp MJ, van Gool JD, Bruinese HW, Kriek R. Early fetal leg movements in myelomeningocele. Lancet 1986;1(8486):917-8. 
23. Sival DA, Begeer JH, Staal-Schreinemachers AL, et al. Perinatal motor behaviour and neurological outcome in spina bifida aperta. Early Hum Dev 1997;50:27-37.

24. Sival DA, Guerra M, den Dunnen WF, et al. Neuroependymal denudation is in progress in full-term human foetal spina bifida aperta. Brain Pathol 2011;21:163-79.

25. McLone DG, Dias MS. The Chiari II malformation: cause and impact. Childs Nerv Syst 2003;19:540-50.

26. Paek BW, Farmer DL, Wilkinson CC, et al. Hindbrain herniation develops in surgically created myelomeningocele but is absent after repair in fetal lambs. Am J Obstet Gynecol 2000;183:1119-23.

27. Deprest, J, Luks F, Lewi L, et al. Minimally invasive procedures rekindle invasive fetal therapy. In: Reproductive Medicine in the Catholic World: a silent schism? I Brosens, Ed. Peeters Publishers, 2006:217-241.

28. Ovaere C, Eggink A, Richter J, et al. Prenatal diagnosis and patient preferences in patients with neural tube defects around the advent of fetal surgery in Belgium and Holland. Fetal Diagn Ther 2015;37:226-34.

29. Moldenhauer JS, Soni S, Rintoul NE, et al. Fetal myelomeningocele repair: the postMOMS experience at the Children's Hospital of Philadelphia. Fetal Diagn Ther 2015;37:235-40.

30. Cohen AR, Couto J, Cummings JJ, et al. Position statement on fetal myelomeningocele repair. Am J Obstet Gynecol 2014;210:107-11.

31. Kohl T. Percutaneous minimally-invasive fetoscopic surgery for spina bifida aperta Part I: Surgical technique and perioperative outcome. Ultrasound Obstet Gynecol 2014;

32. Gregoir C, Engels AC, Gomez O, et al. Fertility, pregnancy and gynecological outcomes after fetoscopic surgery for congenital diaphragmatic hernia. Hum Reprod 2016;31:2024-30.

33. Danzer $\mathrm{E}$, Thomas NH, Thomas AT, et al.Long-term neurofunctional outcome, executive functioning, and behavioral adaptive skills following fetal myelomeningocele surgery. Am J Obstet Gynecol 2016;214:269.e1-8.

34. Papanna R, Fletcher S, Moise KJ, et al. Cryopreserved human umbilical cord for in utero myeloschisis repair. Obstet Gynecol 2016;128:325-30.

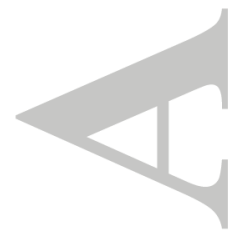

This article is protected by copyright. All rights reserved. 
Table 1: Maternal and fetal outcome data comparing endoscopic (ENDO) and open (OPEN) fetal repair.

\begin{tabular}{|c|c|c|c|}
\hline Data: mean +/- standard deviation or $n(\%)$ & $\begin{array}{l}\text { ENDO } \\
(n=10)\end{array}$ & $\begin{array}{l}\text { OPEN } \\
(\mathrm{n}=20)\end{array}$ & $\begin{array}{l}P \\
\text { value }\end{array}$ \\
\hline Fetal bradycardia intraoperative & $0 / 10(0 \%)$ & $2 / 20(10 \%)$ & 0.8 \\
\hline Chorion-amnion separation & $2 / 9(22 \%)$ & $2 / 20(10 \%)$ & 0.38 \\
\hline Postoperative oligohydramnios & $3 / 10(30 \%)$ & $4 / 20(20 \%)$ & 0.54 \\
\hline Gestational Age (GA) at delivery (weeks) & $36.3+/-2.9$ & $33.8+/-3.7$ & 0.12 \\
\hline $\begin{array}{l}\text { Preterm Premature Rupture of Membranes } \\
\text { (PPROM) }\end{array}$ & 2/9 (22\%) & 7/20 (35\%) & 0.49 \\
\hline Interval: Fetal surgery to delivery (weeks) & $11.5+/-2.8$ & $9.4+/-4.2$ & 0.24 \\
\hline Upper Segment Hysterotomy & $0 / 10(0 \%)$ & $\begin{array}{l}20 / 20 \\
(100 \%)\end{array}$ & 0.00 \\
\hline Vaginal Delivery & $\begin{array}{l}3 / 7(43 \%) * 3 \text { cases undelivered as of } \\
8 / 8 / 2015\end{array}$ & $0 / 20(0 \%)$ & 0.002 \\
\hline Birth Weight (g) & $2562+/-657$ & $\begin{array}{l}2344+/- \\
640\end{array}$ & 0.51 \\
\hline $\begin{array}{l}\text { Maternal Length of Stay after fetal surgery } \\
\text { (LOS) }\end{array}$ & $5+/-1$ & $7+/-3$ & 0.19 \\
\hline Neonatal LOS after delivery: median [range] & $6[2-31]$ & $7[2-51]$ & 0.9 \\
\hline
\end{tabular}


Figure 1: Schematic drawing of neurosurgical closure following dissection and untethering, either (a) through multilayered repair involving the dura, musculofascial structures and skin; or using (b) a dural or (c) skin substitute. In some reports two grafts are used to assist myofascial and skin closure (12). Reproduced, with permission from the University Hospitals Leuven and Myrthe Boymans, artist.

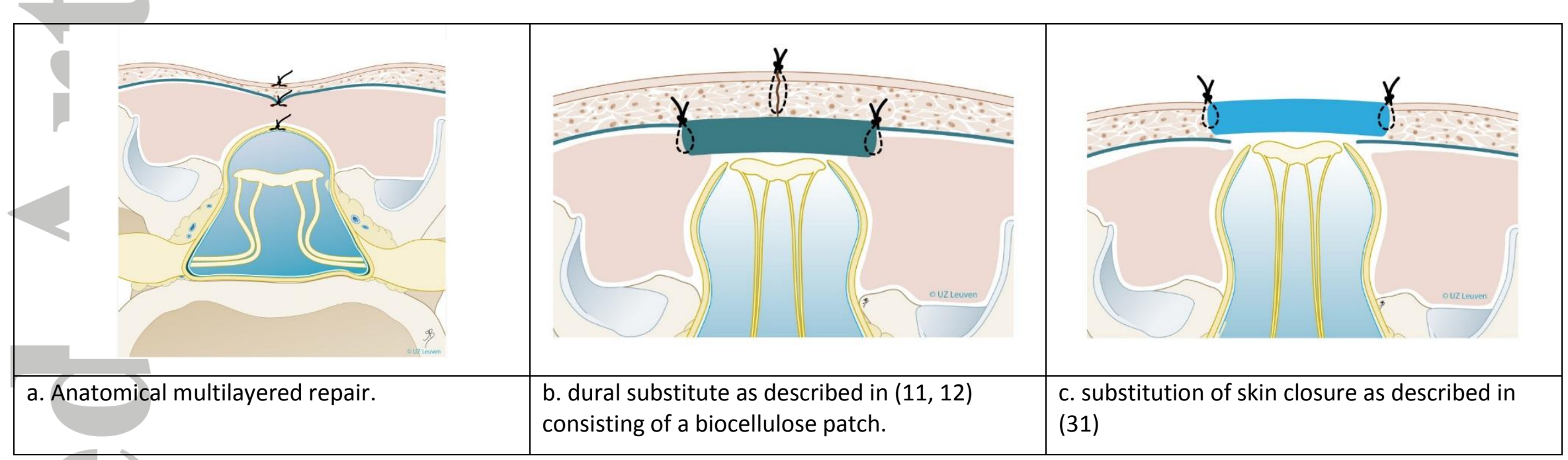

This article is protected by copyright. All rights reserved. 